\begin{tabular}{|c|l|}
\hline Title & On anisotropy and curvature effects for growing crystals \\
\hline Author(s) & Kobay ashi, R.; Giga, Y. \\
\hline Citation & Hokkaido University Preprint Series in Mathematics, 506, 1-38 \\
\hline Issue Date & 2000-12-1 \\
\hline DOI & 10.14943/83652 \\
\hline Doc URL & http://hdl.handle.net/2115/69256 \\
\hline Type & bulletin (article) \\
\hline File Information & pre506.pdf \\
\hline
\end{tabular}

Instructions for use 


\section{On Anisotropy and Curvature Effects for Growing Crystals \\ - In Memory of Prof. Yamaguti - \\ R. Kobayashi and Y. Giga}

Series \#506. December 2000 


\section{HOKKAIDO UNIVERSITY PREPRINT SERIES IN MATHEMATICS}

\#482 N. H. Bingham and A. Inoue, Tauberian and Mercerian theorems for systems of kernels, 16 pages. 2000.

\#483 N. H. Bingham and A. Inoue, Abelian, Tauberian and Mercerian theorems for arithmetic sums, 29 pages. 2000 .

\#484 I. A. Bogaevski and G. Ishikawa, Lagrange mappings of the first open Whitney umbrella, 22 pages. 2000.

\#485 A. Arai and H. Kawano, A class of deformations of the Schrödinger representation of the Heisenberg commutation relation and exact solution to a Heisenberg equation and a Schrödinger equation, 22 pages. 2000.

\#486 T. Nakazi, Functions in $N_{+}$with the positive real parts on the boundary, 21 pages. 2000.

\#487 Y. Shibukawa, Classification of the $R$-operator, 36 pages. 2000.

\#488 A. Inoue, Asymptotic behaviour for partial autocorrelation functions of fractional ARIMA processes, 20 pages. 2000 .

\#489 S. Ohtani, Construction of unramified Galois extensions over maximal abelian extensions of algebraic number fields, 14 pages. 2000.

\#490 T. Nakazi and T. Yamamoto, The real part of an outer function and a Helson-Szegö weight, 13 pages. 2000 .

\#491 A. Yamagami, On Gouvêás conjecture on controlling the conductor, 11 pages. 2000.

\#492 I. Tsuda and M. Hatakeyama, Making sense of internal logic: Theory and a case study, 10 pages. 2000.

\#493 I. Tsuda, Towards an interpretation of dynamic neural activity in terms of chaotic dynamical systems, 73 pages. 2000.

\#494 T. Mikami, Optimal control for absolutely continuous stochastic processes and the mass transportation problem, 17 pages. 2000 .

\#495 M. Arisawa and Y. Giga, Anisotropic curvature flow in a very thin domain, 21 pages. 2000.

\#496 T. Nakazi, Backward shift invariant subspaces in the bidisc, 9 pages. 2000.

\#497 Y. Giga, K. Inui, J. Kato and S. Matsui, Remarks on the uniqueness of bounded solutions of the NavierStokes equations, 4 pages. 2000.

\#498 Y. Giga, M. Paolini and P. Rybka, On the motion by singular interfacial energy, 21 pages. 2000.

\#499 J. Escher and Y. Giga, On a limiting motion and self-interactions of curves moved by the intermediate surface diffusion flow, 12 pages. 2000.

\#500 I. Tsuda and S. Kuroda, Cantor coding in the hippocampus, 20 pages. 2000.

\#501 M. Tsujii, Fat solenoidal attractors, 20 pages. 2000.

\#502 A. Arai, Ground state of the massless Nelson model without infrared cutoff in a non-Fock representation, 19 pages. 2000.

\#503 Y. Giga, S. Matsui and O. Sawada, Global existence of two-dimensional Navier-Stokes flow with nondecaying initial velocity, 19 pages. 2000 .

\#504 A. Inoue and Y. Kasahara, Partial autocorrelation functions of the fractional ARIMA processes with negative degree of differencing, 14 pages. 2000.

\#505 T. Nakazi, Interpolation problem for $\ell^{1}$ and a uniform algebra, 12 pages. 2000. 


\title{
On Anisotropy and Curvature Effects for Growing Crystals
}

- In Memory of Prof. Yamaguti -

\author{
R.Kobayashi ${ }^{1}$ and Y.Giga ${ }^{2}$ \\ Research Institute for Electronic Science \\ Hokkaido University, Sapporo 060, Japan ${ }^{1}$ \\ Department of Mathematics \\ Hokkaido University, Sapporo 060, Japan ${ }^{2}$
}

November 30, 2000

\begin{abstract}
We will present an introductory review of the mathematics for investigating the interfacial motion in crystal growth problems. Anisotropy is quite an important factor in such problems. There are two types of anisotropy - the kinetic anisotropy and the one of curvature effect. The main theme of this article is how the kinetic anisotropy determines the growth form of the crystal and how the curvature effect works on it.
\end{abstract}




\section{Introduction}

In this article the equation of the form

$$
V=\mu(\boldsymbol{n})\left(c-\kappa_{\gamma}\right)
$$

is investigated to study the behavior of growing crystals. This equation is derived under several physical assumptions. Crystals usually grow in some diffusion field of materials and/or temperature field that might be a rate-determining process of crystal growth. However, it is not the case here, and the circumstances surrounding the crystal is assumed to be uniform. The driving force for crystallization is expressed by the sum of chemical potentials which are derived from bulk free energy and surface free energy. The driving force from bulk is considered to be a constant $c$ by the uniformity of surroundings, also $c$ is taken to be positive since we concentrate on the growth problem. On the other hand, the driving force from surface depends on the geometric information written in the form of weighted mean curvature $\kappa_{\gamma}$. Weighted mean curvature is an extension of the usual mean curvature when the surface free energy $\gamma$ depends on the surface orientation. At last, it is assumed that the growth rate $V$ is proportional to the driving force and the sensitivity $\mu$ is orientation dependent, which we call a kinetic anisotropy. Therefore the equation (1.1) includes two types of anisotropy, anisotropy of surface free energy and kinetic anisotropy. It is by now well known that the first order approximation of the shape of the growing crystal is determined by kinetic anisotropy. However, it is often important to understand the effect of curvature. We shall distinguish several phenomena which appear to be the same but is different.

This paper is organized as follows. In the section 2, the basics of related mathematics will be presented. Then we will demonstrate how the solution of the equation without curvature effect behaves in the section 3 . In the last section, the equation (1.1) will be 
studied under several special situations. This paper is characterized as an introduction to the theoretical study of crystal growth and related mathematics. In order to make this paper understandable for non-mathematicians, we present various examples of typical interfacial motions induced by (1.1) instead of developing a general and rigorous theory. We will give some comments on related introductory references at the end of each section if necessary. We have no intension to exhaust the references.

\section{Memories of Prof. Yamaguti (by Kobayashi)}

There certainly exists a mathematicians' group called "Yamaguti school", although it is not so strictly defined. I think Prof. Yamaguti did not like to make things too strict, and of course, to make a closed group of his followers. He never imposed borders that limit our way to do mathematics. As far as I remember, I never heard he's saying "You should do it in such and such way ...". I am an applied mathematician who never (or seldom) prove theorems, that is my style. I might have a harder time to live with such a style if I belong to another mathematicians' community. On the contrary, there was no problem at all, people in Yamaguti school including Prof. Yamaguti himself encouraged me to find my own way. Thus I really thank him for his open-minded soul and all the people who inherit it in their hearts.

Recently I found a statement about a definition of good leaders in some book. It says that the good leader is a person who could make the followers not only respect him/her but also think themselves to be indispensable to their party. Yes, that's true. Everybody knows Prof. Yamaguti is the very man who satisfies that condition of successful leaders. He seemed to behave not so intentionally to encourage his students or disciples. Rather that was a result of his virtue by nature, I believe. And I find that it's almost impossible to have such a type of leader as "Yamaguti" after him. 


\section{Basic Mathematics}

In this section, we introduce mathematical concepts and tools needed in the subsequent sections. All the problems in this article will be considered in the 2 or 3 dimensional space. We will express the 2 dimensional space and the 3 dimensional space by the same notation $R^{n}$ unless we need to specify the spatial dimension.

\subsection{PPHD1 Functions}

If the function $f(n)$ is defined on the unit sphere $S^{n-1}$, i.e., $f$ is a function of orientation, it can be extended to the whole space $R^{n}$ by putting

$$
f(x)=|x|_{2} f\left(\frac{x}{|x|_{2}}\right) \text { for } \boldsymbol{x} \neq \mathbf{0} \quad \text { and } \quad f(x)=0 \text { for } \boldsymbol{x}=\mathbf{0}
$$

where $|x|_{2}=\left(\sum_{i} x_{i}^{2}\right)^{1 / 2}$ for $x=\left(x_{1}, \ldots, x_{n}\right)$. We will use the same notation $f$ for the extended function. This extended function $f$ satisfies

$$
f(\lambda x)=\lambda f(x) \quad \text { for } \lambda \geq 0 \text { and } x \in R^{n} .
$$

A function which satisfies (2.3) is called a PHD1 (positively homogeneous of degree 1) function. In this article, we handle the positive PHD1 functions only, and use the notation PPHD1 function for the positive PHD1 function hereafter. (Positive means taking positive values except at the origin.) Fig.2.1 shows graphs of PPHD1 functions defined on $R^{2}$, which are wrinkled cones in general. Note that the slope of the generating line of the cone corresponding to the direction $\boldsymbol{n}$ equals to $f(\boldsymbol{n})$.

As is seen in Fig.2.1, PPHD1 functions are not differentiable at the origin. Assuming differentiability of a PPHD1 function $f$ except at the origin, we have a vector-valued function $\nabla f(\boldsymbol{x})$ for $\boldsymbol{x} \neq \mathbf{0}$. Then the following properties immediately follow. (See 

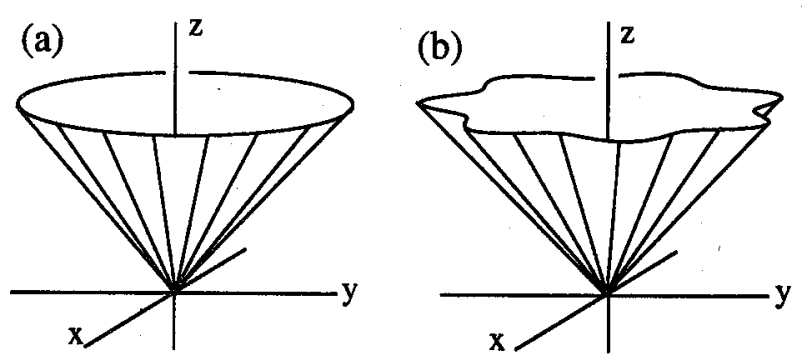

Figure 2.1: (a) Graph of an isotropic PPHD1 function $z=f(x, y)$ (b) Graph of an anisotropic PPHD1 function $z=f(x, y)$

Appendix 1.)

$$
\begin{aligned}
& \nabla f(\lambda \boldsymbol{x})=\nabla f(\boldsymbol{x}) \\
& f(\boldsymbol{x})=\boldsymbol{x} \cdot \nabla f(\boldsymbol{x}) \\
& \boldsymbol{x} \cdot d \nabla f(\boldsymbol{x})=0
\end{aligned}
$$

Equation (2.4) tells us that $\nabla f$ essentially depends only on the orientation. From (2.5) it follows that $f(\boldsymbol{n})=\boldsymbol{n} \cdot \nabla f(\boldsymbol{n})$, therefore the radial component of $\nabla f$ is always $f(\boldsymbol{n}) \boldsymbol{n}$. The expression $d \nabla f(\boldsymbol{x})$ means a small displacement of $\nabla f(\boldsymbol{x})$ against a small displacement $d \boldsymbol{x}$ from the position $\boldsymbol{x}$, and it is rigorously defined as an exterior derivative of $\nabla f(\boldsymbol{x})$.

\section{Norms}

All the norms on $R^{n}$ are PPHD1 functions. Among them, the 2-norm $|x|_{2}=$ $\left(\sum_{i} x_{i}^{2}\right)^{1 / 2}$ is a special norm since it gives an isotropic PPHD1 function, i.e., $f(\boldsymbol{n}) \equiv 1$ on $S^{n-1}$. For this $f, \nabla f(\boldsymbol{x})=\frac{\boldsymbol{x}}{|\boldsymbol{x}|_{2}}$ and it has only a radial component. The tangential component of $\nabla f$ (the component perpendicular to the radial one) is non-zero only when $f$ is anisotropic.

\section{Two Dimensional Problems}

If we treat 2 dimensional problems, it is convenient to adopt the polar coordinate 
system $(r, \theta)$. A positive function defined on $S^{1}$ can be expressed by the $2 \pi$-periodic positive function $f_{0}(\theta)$. Then it is extended to the PPHD1 function as

$$
f(\boldsymbol{x})=r f_{0}(\theta)
$$

Here we distinguish the notation $f_{0}$ and $f$ to avoid confusion. Then $\nabla f$ is a function of $\theta$ alone by the property (2.4) and is actually expressed by

$$
\nabla f=f_{0}(\theta) \boldsymbol{e}_{r}(\theta)+f_{0}^{\prime}(\theta) \boldsymbol{e}_{\theta}(\theta)
$$

where $e_{r}(\theta)=(\cos \theta, \sin \theta)^{T}$ and $e_{\theta}(\theta)=(-\sin \theta, \cos \theta)^{T}$. A simple calculation gives a formula

$$
d \nabla f=\left(f_{0}(\theta)+f_{0}^{\prime \prime}(\theta)\right) e_{\theta}(\theta) d \theta
$$

All the examples in this article will be given in the form (2.7).

\subsection{Convexity}

As stated above, all norms are PPHD1 functions. In addition, norms satisfy the triangle inequality (or subadditive property)

$$
f(\boldsymbol{x}+\boldsymbol{y}) \leq f(\boldsymbol{x})+f(\boldsymbol{y})
$$

while it is not always valid for general PPHD1 functions. However whether the inequality holds or not is quite an important property for PPHD1 functions - convexity. The function defined on $R^{n}$ is convex if

$$
f((1-\lambda) \boldsymbol{x}+\lambda \boldsymbol{y}) \leq(1-\lambda) f(\boldsymbol{x})+\lambda f(\boldsymbol{y}) \text { for } \boldsymbol{x}, \boldsymbol{y} \in R^{n} \text { and } \lambda \in[0,1]
$$

If the function $f$ is a PPHD1 function, the following three properties are equivalent. (See Appendix 2.) 
(1) The function $f$ is convex.

(2) The function $f$ satisfies triangle inequality (or $f$ is subadditive).

(3) The set $\left\{\boldsymbol{x} \in R^{n} ; f(\boldsymbol{x}) \leq 1\right\}$ is convex.

\section{Convexification}

For a non-convex function $f$, the unique convex function $\bar{f}$ called convexification of $f$ is defined as follows.

$$
\begin{aligned}
\bar{f}(\boldsymbol{x}) & =\inf \left\{\sum_{i} \lambda_{i} f\left(\boldsymbol{x}_{i}\right) ; \quad \boldsymbol{x}=\sum_{i} \lambda_{i} \boldsymbol{x}_{i}, \sum_{i} \lambda_{i}=1 \text { and } \lambda_{i} \geq 0\right\} \\
& =\sup \{g(\boldsymbol{x}) ; g \text { is convex and } g \leq f\}
\end{aligned}
$$

i.e., it is the greatest of all the convex functions no greater than $f$. It is, so to say, obtained by filling up the concave part of the non-convex function like the convex hull is constructed from the non-convex set. Actually these two are closely related, say, the set $\left\{x \in R^{n} ; \bar{f}(\boldsymbol{x}) \leq 1\right\}$ coincide with a convex hull of the set $\left\{x \in R^{n} ; f(x) \leq 1\right\}$.

\subsection{Graphical constructions}

There are several graphical methods to examine the properties of PPHD1 functions. We will demonstrate what they mean and how they are related. The first one is a radial plot of $f$ expressed by

$$
C_{f}=\left\{f(\boldsymbol{n}) \boldsymbol{n} ; \boldsymbol{n} \in S^{n-1}\right\}
$$

The second is a radial plot of the reciprocal of $f$ which is also, as easily seen, an iso-surface (level set) of $f$ given by

$$
F_{f}=\left\{\frac{1}{f(n)} n ; n \in S^{n-1}\right\}=\left\{x \in R^{n} ; \quad f(x)=1\right\} .
$$

This is called Frank plot when $f(\boldsymbol{n})$ is taken to be $\gamma(\boldsymbol{n})$ which evaluate the surface free energy density of the crystal. If PPHD1 function $f$ is continuous, both of $C_{f}$ and $F_{f}$ 
are surfaces or closed curves surrounding the origin, and they are smooth if $f$ is smooth. A closed set enclosed by $F_{f}$ is called Frank diagram and denotes $\tilde{F}_{f}$, i.e., $\tilde{F}_{f}=\{\boldsymbol{x} \in$ $\left.R^{n} ; f(\boldsymbol{x}) \leq 1\right\}$. The third is a gradient plot of $f$,

$$
\Xi_{f}=\left\{\nabla f(\boldsymbol{n}) ; \quad \boldsymbol{n} \in S^{n-1}\right\}
$$

In the case $f=\gamma, \nabla f$ is called $\xi$-vector or Cahn-Hoffman vector[13, 2]. At this point we impose differentiability on $f$ although the definition of $\nabla f$ will be extended in order to apply it to the cusp-shaped $f$ which is common in the crystal problems. It will be discussed later. The last one is called Wulff set (Wulff shape) in the context of surface free energy, which is defined as follows:

$$
W_{f}=\left\{\boldsymbol{x} \in R^{n} ; \boldsymbol{n} \cdot \boldsymbol{x} \leq f(\boldsymbol{n}) \text { for all } \boldsymbol{n} \in S^{n-1}\right\}
$$

The boundary $\partial W_{f}$ is an inner envelope of planes (or lines) perpendicular to $\boldsymbol{n}$ and passing through $f(\boldsymbol{n}) \boldsymbol{n}$, which we call $W$ ulff plot. By definition $W_{f}$ is always convex regardless of the convexity of $f$. There are several useful notions in convex analysis to describe the Wulff set, which are shown in Appendix 3.

Let us demonstrate these graphical methods by the examples. Consider the 2 dimensional space and take the form $f_{0}(\theta)=1-\alpha \cos 4 \theta$ in (2.7) and draw all the types of graphs for it.

\section{Example 1}

Fig2.2 displays graphs of the convex function which is obtained by setting $\alpha$ a small positive value. The first panel shows $C_{f}$ - radial plot of $f$. The second panel displays $F_{f}$ which is a radial plot of $f^{-1}$. The vector $\nabla f(\boldsymbol{n})$ is normal to $F_{f}$ at the point $\frac{1}{f(\boldsymbol{n})} \boldsymbol{n}$ since $F_{f}$ is a contour plot of $f$ and $\nabla f$ depends only on the orientation. $\Xi_{f}$ in the third panel is a plot of $\nabla f(\boldsymbol{n})$ given by the form (2.8). The normal vector of $\Xi_{f}$ at the point $\nabla f(n)$ is $n$ itself because of the property (2.6). The figure in the fourth panel is drawn 

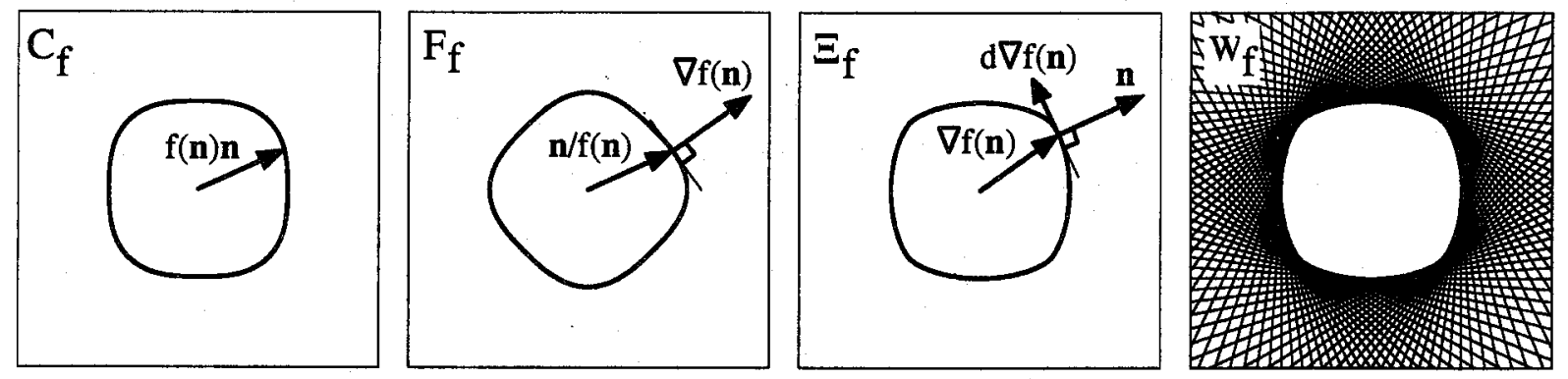

Figure 2.2: Graphs of a convex function given by $f=r(1-\alpha \cos 4 \theta)$ with $\alpha=0.05$.

by somewhat indirect way. $W_{f}$ is a region inside of the inner envelope formed by the lines indicated. In this example, $\partial W_{f}$ exactly coincides with $\Xi_{f}$, which is not necessarily true as will be shown in the next example.

\section{Example 2}

If the parameter $\alpha$ becomes larger, the function $f$ will lose convexity. Actually convexity is lost when the local convex condition $f_{0}(\theta)+f_{0}^{\prime \prime}(\theta) \geq 0$ is violated for some $\theta$, which means $d \nabla f(\theta)$ changes direction somewhere. Thus the critical value is given by $\alpha_{c}=1 / 15$ in this example. Fig.2.3 indicates graphs of a non-convex function. If the function $f$ becomes non-convex, the new features emerge in the graphs. The region surrounded by $F_{f}$ (i.e., Frank diagram $\tilde{F}_{f}$ ) becomes a non-convex set and $\Xi_{f}$ is having "ears" and is no more a simple closed curve. $W_{f}$ becomes to have corners although $f$ is
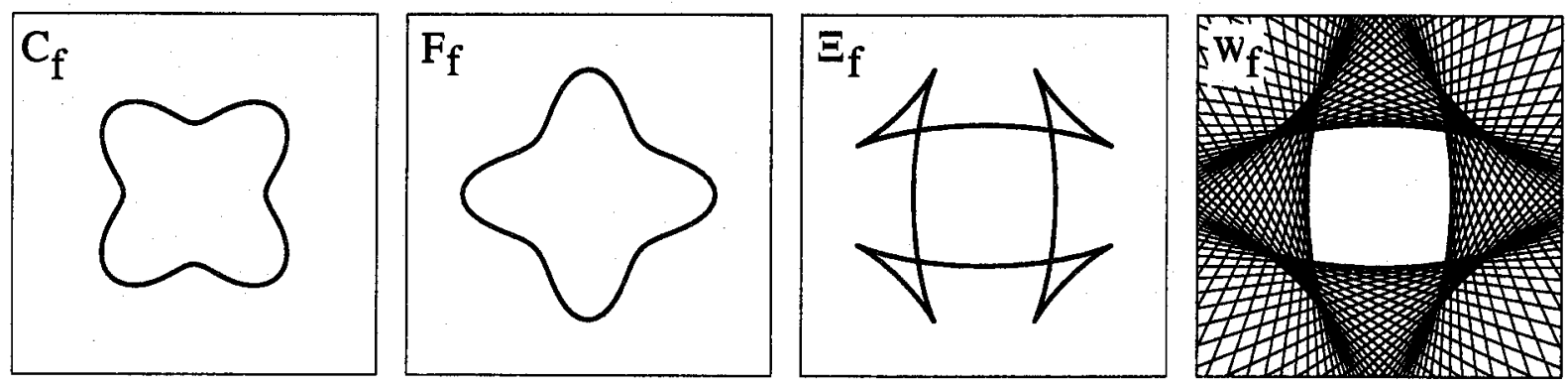

Figure 2.3: Graphs of a non-convex function given by $f=r(1-\alpha \cos 4 \theta)$ with $\alpha=0.25$. 
completely smooth, and $W_{f}$ is nothing more than the region which is surrounded by the curves obtained by cutting off the ears from $\Xi_{f}$. Although $W_{f}$ itself has no information about ears, we can observe in the fouth panel of Fig. 2.3 the switchback of the lines to form the shadow of ears outside of $W_{f}$.

There is a canonical mapping $J: \boldsymbol{n} / f(\boldsymbol{n}) \longrightarrow \nabla f(\boldsymbol{n})$ which maps $F_{f}$ onto $\Xi_{f}$ (see Fig.2.2). It is not difficult to prove that $\partial W_{f}=\Xi_{f}$ if $\tilde{F}_{f}$ is strictly (in the sense of curvature) convex and smooth. In this case the map $J$ is a diffeomorphism from $F_{f}$ to $\partial W_{f}$.

\section{Example 3}

Let us see next how the convexification changes all these graphs. The original function $f$ is the same with the previous example and the corresponding plots $C_{f}, F_{f}$ and $\Xi_{f}$ are drawn by dashed curves as shown in Fig.2.4. The plain curves are plots for $\bar{f}$ which is a convexified $f . F_{\vec{f}}$ is obtained by the common tangent construction from $F_{f}$, where the common tangent implies common gradient through the angle range I. Then $\nabla \bar{f}(\boldsymbol{n})$ stays at the corner point $\mathrm{P}$ of $\Xi_{\bar{f}}$ when $\boldsymbol{n}$ run through the range $\mathrm{I}$, consequently the ear is removed. It is now clear that the ears in $\Xi_{f}$ correspond to the concave parts of $F_{f}$ which do not appear in $F_{\bar{f}}$. $W_{\bar{f}}$ always coincide with $W_{f}$ (see Appendix 3), which means different
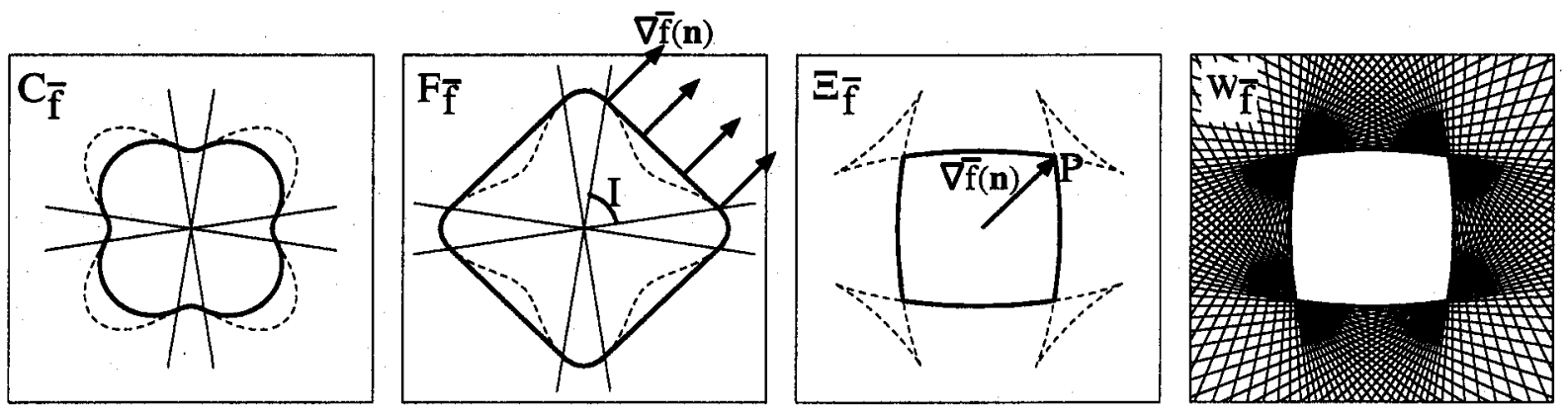

Figure 2.4: Graphs of $\bar{f}$ which is a convexification of $f=r(1-\alpha \cos 4 \theta)$ with $\alpha=0.25$. 
$f$ 's can give the same $W_{f}$. In other words, $W_{f}$ does not have all the information of $f$, actually it exactly loses the information of the concave part (range I, for example) of $f$. It is essential that $\partial W_{f}=\Xi_{f}$ if and only if $f$ is convex. Note that the region surrounded by $C_{f}$ is not necessarily convex if $f$ is convex as is seen in this example.

\subsection{Extension of $\nabla f$}

We will consider the anisotropic normal velocity $v(\boldsymbol{n})$ of the crystalline surface in the next section. The velocity function $v(\boldsymbol{n})$ often has a non-differentiable profile which gives inward cusps to its radial plot. Therefore we extend the definition of $\nabla f$ for such a special class of $f$.

Here we will restrict ourselves to the two dimensional problems for simplicity, and refer to the three dimensional ones in the end of this subsection shortly. As stated previously, $f$ is written in the form $f(\boldsymbol{x})=r f_{0}(\theta)$. If $C_{f}$ has a inward cusp at $\theta_{0}, f_{0}^{\prime}(\theta)$ is discontinuous at $\theta_{0}$, while $f_{0}(\theta)$ is a continuous function. Here we assume that the limits $f_{0}^{\prime}\left(\theta_{0} \pm 0\right)$ exist, then $\nabla f\left(\cos \left(\theta_{0} \pm 0\right), \sin \left(\theta_{0} \pm 0\right)\right)=f_{0}\left(\theta_{0}\right) \boldsymbol{e}_{r}\left(\theta_{0}\right)+f_{0}^{\prime}\left(\theta_{0} \pm 0\right) \boldsymbol{e}_{\theta}\left(\theta_{0}\right)$ from (2.8). At such a cusp point $\nabla f$ cannot be defined in usual sense. The extended $\nabla f$ at the cusp point is defined as a set of vectors by

$$
\begin{aligned}
\nabla f & =\left\{(1-\lambda) \nabla f\left(\cos \left(\theta_{0}-0\right), \sin \left(\theta_{0}-0\right)\right)+\lambda \nabla f\left(\cos \left(\theta_{0}+0\right), \sin \left(\theta_{0}+0\right)\right) ; \quad 0 \leq \lambda \leq 1\right\} \\
& =\left\{f_{0}\left(\theta_{0}\right) \boldsymbol{e}_{r}\left(\theta_{0}\right)+\left((1-\lambda) f_{0}^{\prime}\left(\theta_{0}-0\right)+\lambda f_{0}^{\prime}\left(\theta_{0}+0\right)\right) \boldsymbol{e}_{\theta}\left(\theta_{0}\right) ; 0 \leq \lambda \leq 1\right\} .
\end{aligned}
$$

Note that the radial component of the vectors belonging to the extended $\nabla f$ at the cusp are all the same. Corresponding to the extended $\nabla f$, there appears a line segment in $\Xi_{f}$ and $W_{f}$ which we will call a facet in this article.

\section{Example 4}

Let us take $f_{0}(\theta)=1+\alpha\left(|\cos 2 \theta|-\frac{1}{2}\right)$. Fig.2.5 gives an example in which $C_{f}$ has 
shallow cusps. $F_{f}$ has outward corners corresponding to these cusps. If such cusps are not so deep or equivalently corners are not so pointed, $f$ can be convex and thus facets
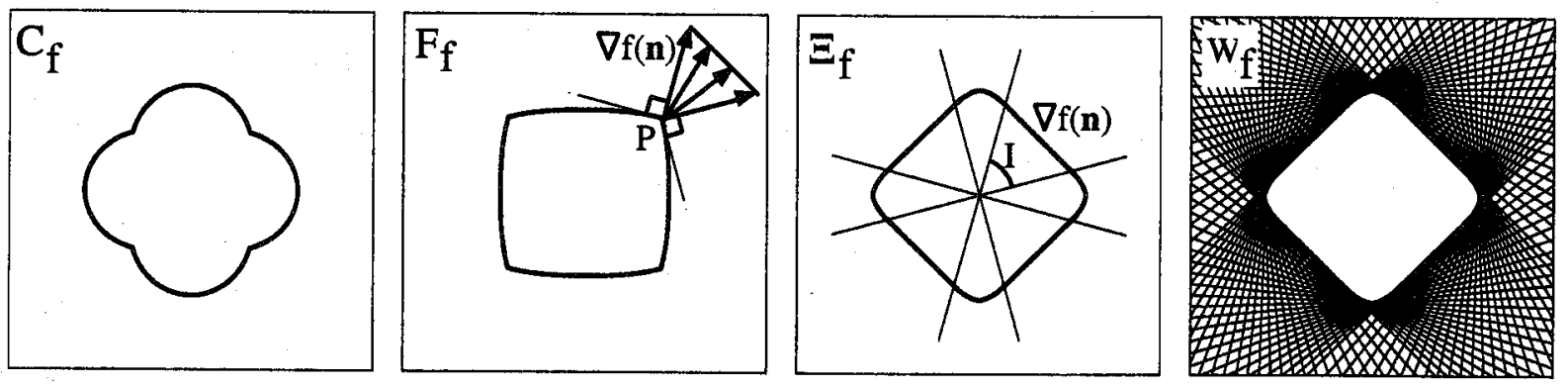

Figure 2.5: Graphs of a non-differentiable convex function given by $f=r\{1+\alpha(|\cos 2 \theta|-$ $1 / 2)\}$ with $\alpha=0.25 . \partial W_{f}$ is partially faceted.

and curved segment coexist in $\Xi_{f}\left(=\partial W_{f}\right)$. The line segment which corresponds to an extended $\nabla f$ at the corner point $\mathrm{P}$ appears as a facet with the angle range $\mathrm{I}$ in $\Xi_{f}$

\section{Example 5}

Fig.2.6 presents graphs in which the cusps of $C_{f}$ are deep. In such a case, corners are pointed enough to make $F_{f}$ non-convex. Thus $\Xi_{f}$ has ears, and the square is remained after removing ears from it, which implies that $\partial W_{f}$ is fully faceted.
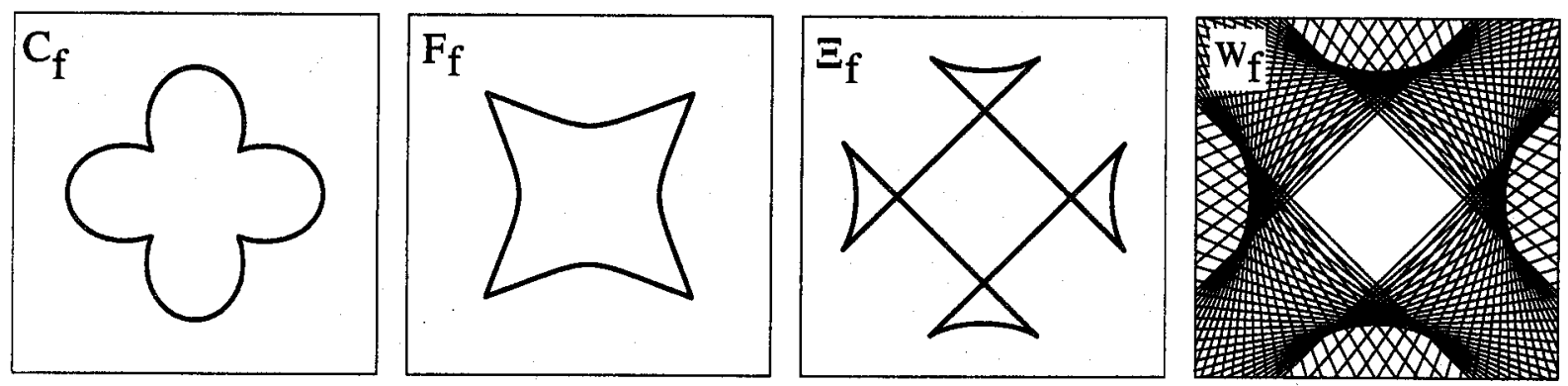

Figure 2.6: Graphs of a non-differentiable non-convex function given by $f=r\{1+$ $\alpha(|\cos 2 \theta|-1 / 2)\}$ with $\alpha=0.70 . \partial W_{f}$ is a fully faceted square. 


\section{Example 6}

One more example is given by $f_{0}(\theta)=1+\alpha\left(|\cos 2 \theta|-\frac{1}{2}\right)+\beta\left(|\sin 2 \theta|-\frac{1}{2}\right)$. It is easily seen that $\partial W_{f}$ is a fully faceted octagon if the values of $\alpha$ and $\beta$ are close and not so small. By choosing $\alpha$ and $\beta$ appropriately, $\partial W_{f}$ can be a fully faceted square as well
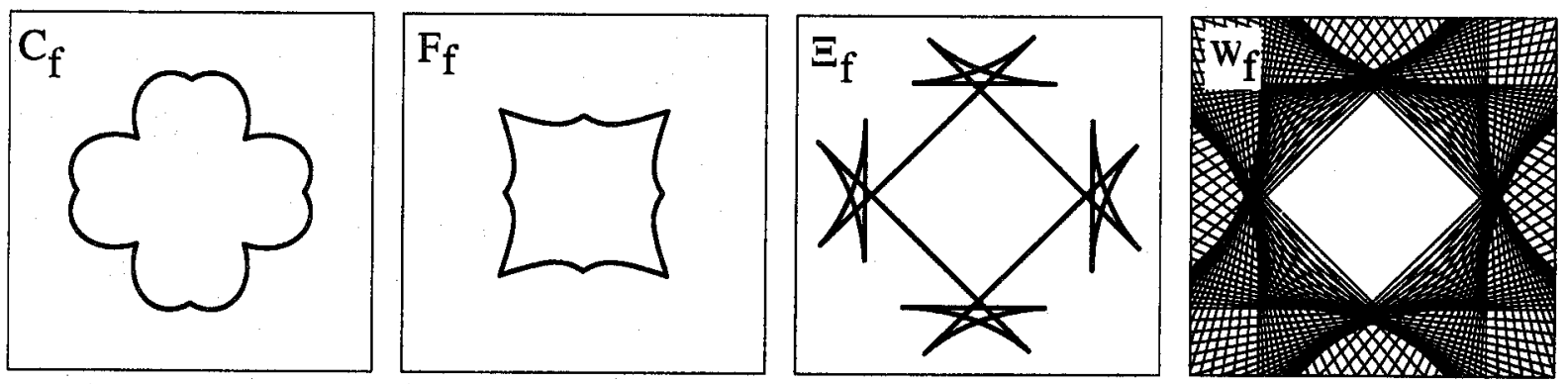

Figure 2.7: Graphs of a non-differentiable non-convex function given by $f=r\{1+$ $\alpha(|\cos 2 \theta|-1 / 2)+\beta(|\sin 2 \theta|-1 / 2)\}$ with $\alpha=0.40$ and $\beta=0.80$. $\partial W_{f}$ is a fully faceted square (not an octagon):

as the previous example as shown in Fig.2.7. The difference from the previous one is the existence of hidden facet induced by the shallower cusps, which will be discussed in the next section.

\section{Extension of $\nabla f$ in the 3 dimensional space}

In the 3 dimensional space, it is somewhat messy to define the extended $\nabla f$ at nondifferentiable point by the similar way to the 2 dimensional case using the interpolation of limiting values of $\nabla f$. It is given by the differentiation of Clarke [5] which is an extension of subdifferentials of convex functions.

Note The readers are referred to the article [1] for physical interpretation of convexity as well as an introduction to graphical constructions. 


\section{Anisotropic motion without curvature effect}

In this section, we will study the following simple equation which describes an anisotropic motion of crystalline surface,

$$
V=v(\boldsymbol{n})
$$

where $V$ is a normal velocity of the surface of crystal and $\boldsymbol{n}$ is an outer normal unit vector at the interface. The curvature effect is ignored here. Such a treatment is reasonable if we are interested in large crystals and do not care much about a small scale structure at corners or edges of the crystal. The velocity function $v(\boldsymbol{n})$ is assumed to be positive, and is extended to the PPHD1 function still denoted by $v$.

\subsection{Equilibrium Forms and Asymptotic Growth Forms}

It should be noted here that there is a mathematical similarity between the equilibrium form of the crystal and the asymptotic growth forms.

\section{Equilibrium Form}

When $\gamma(\boldsymbol{n})$ represents the surface free energy density of the crystalline surface whose outer normal is $\boldsymbol{n}$. Equilibrium form of the crystal is a form which minimize the total surface free energy $\int_{S} \gamma(\boldsymbol{n}) d S$ under the constant volume constraint. This problem was solved by Wulff about a hundred years ago, and the answer is a Wulff plot $\partial W_{\gamma}$. Later rigorous proofs were given by various authors. (See the review paper [26] and the book [12].)

\section{Asymptotic Growth Form}

Let us consider an asymptotic growth form of (3.18). Here we define the surface 
$S_{t}^{\text {asym }}$ at the time $t$ by

$$
S_{t}^{a s y m}=t \partial W_{v}=\left\{t \boldsymbol{x} ; \boldsymbol{x} \in \partial W_{v}\right\}
$$

which is a constantly growing Wulff plot. Because $\partial W_{v} \subset \Xi_{v}$, each point on $\partial W_{v}$ is expressed by $\nabla v(\boldsymbol{n})$ where $\boldsymbol{n}$ is a unit vector normal to $\partial W_{v}$ at the point. The relation $v(\boldsymbol{n})=\boldsymbol{n} \cdot \nabla v(\boldsymbol{n})$ guarantees that $S_{t}^{a s y m}$ is a solution of (3.18). When we consider the growth problem, $W_{v}$ and $\partial W_{v}$ are called a kinetic Wulff set (Wulff shape) and a kinetic Wulff plot, respectively. Note that $t \Xi_{v}$ is not a good choice for the solution of (3.18), since $\Xi_{v}$ cannot be a crystalline surface if $v$ is non-convex. In the next subsection, it will be shown that the kinetic Wulff plot $\partial W_{v}$ is actually an asymptotic growth form of (3.18).

In contrast with the problem of equilibrium form, to obtain an asymptotic growth form is not a minimization problem originally. However, it was clarified that the solution is a kinetic Wulff plot $\partial W_{v}$ as well as the equilibrium form is a Wulff plot $\partial W_{\gamma}$ which minimizes $\int_{S} \gamma(\boldsymbol{n}) d S$ under the constant volume constraint. Therefore the kinetic Wulff plot has to be a minimizer of $\int_{S} v(\boldsymbol{n}) d S$ under the constant volume constraint. The quantity $\int_{S} v(\boldsymbol{n}) d S$ is an increment of volume of the crystal per unit time. Thus the kinetic Wulff plot is the form which grows slowest of all the forms. In fact, it is proved by [18] that the quantity $\int_{S} v(\boldsymbol{n}) d S /(\text { volume })^{1-1 / n}$ is strictly decreasing under the evolution $S_{t}\left(\subset R^{n}\right)$ of (3.18) unless $S_{t}$ is an asymptotic Wulff plot $S_{t}^{a s y m}$, in which case the quantity is constant in time. Here "volume" denotes the volume enclosed by $S$.

\subsection{Evolution Process}

In order to solve (3.18) for some appropriate initial surface, we can adopt the method of characteristics. Take the point $\boldsymbol{x}_{0}$ on the initial surface $S_{0}$ and let $\boldsymbol{n}_{0}$ be an outer normal 
unit vector of $S_{0}$ at $\boldsymbol{x}_{0}$. Then the characteristics that starts from $\boldsymbol{x}_{0}$ is given by

$$
\boldsymbol{x}(t)=\boldsymbol{x}_{0}+t \nabla v\left(\boldsymbol{n}_{0}\right)
$$

which is a half straight line parameterized by the time variable $t \geq 0$. Note that $(3.20)$ expresses a bandle of half straight lines which radiate from the point $\boldsymbol{x}_{0}$ when $\nabla v\left(\boldsymbol{n}_{0}\right)$ is not a single vector but a set of vectors resulted from the non-differentiability of $v$. The set $P_{t}$ at time $t \geq 0$ is defined by

$P_{t}=\left\{\boldsymbol{x}(t)=\boldsymbol{x}_{0}+t \nabla v\left(\boldsymbol{n}_{0}\right) ; \boldsymbol{x}_{0} \in S_{0}, \boldsymbol{n}_{0}\right.$ is an outer normal unit vector of $S_{0}$ at $\left.\boldsymbol{x}_{0}\right\}$

At first, we assume that the set $P_{t}$ forms a surface for arbitrary $t \geq 0$. To satisfy this assumption, $v$ must be convex, otherwise $P_{t}$ cannot form a surface for large $t$ since the shape of $P_{t}$ converges to $\Xi_{v}$ when $t$ tends to $+\infty$. Let us confirm that all the area segments of $P_{t}$ along one characteristics are parallel. By (3.20), $d \boldsymbol{x}(t)=d \boldsymbol{x}_{0}+t d \nabla v\left(\boldsymbol{n}_{0}\right)$ holds. Then it follows that $\boldsymbol{n}_{0} \cdot d \boldsymbol{x}(t)$ vanishes from $\boldsymbol{n}_{0} \cdot d \boldsymbol{x}_{0}=0$ and (2.6). Therefore the area segment along the characteristics $\boldsymbol{x}(t)$ are all normal to $\boldsymbol{n}_{0}$, and thus parallel. Thus the normal component of $\frac{d \boldsymbol{x}(t)}{d t}$ is calculated by $\boldsymbol{n}_{0} \cdot \frac{d \boldsymbol{x}(t)}{d t}=\boldsymbol{n}_{0} \cdot \nabla v\left(\boldsymbol{n}_{0}\right)=v\left(\boldsymbol{n}_{0}\right)$, which ensures that $P_{t}$ is a solution of (3.18).

In the following examples, the unit circle will be taken as an initial surface and the function $v$ is assumed to be the same type of function as adopted in the examples in the previous section. Figures will display not $P_{t}$ but its scaled version $\tilde{P}_{t}$ which is defined by $\tilde{P}_{t}=\frac{1}{t+1} P_{t}=\frac{1}{t+1} S_{0}+\frac{t}{t+1} \Xi_{v}$, in order to emphasize an evolution of the shape by ignoring the growth in size. The definition of $\tilde{P}_{t}$ implies that it is an interpolation between the two sets $S_{0}$ and $\Xi_{v}$, thus $\tilde{P}_{0}=S_{0}$ and $\tilde{P}_{+\infty}=\Xi_{v}$.

\section{Example 7}

The function $v$ is the same with $f$ in Example 1 which is convex and differentiable. 
(a)

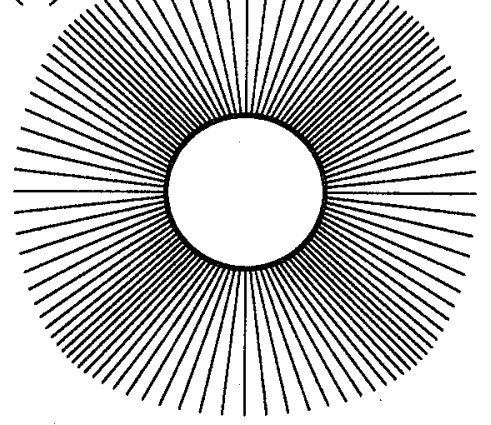

(b)

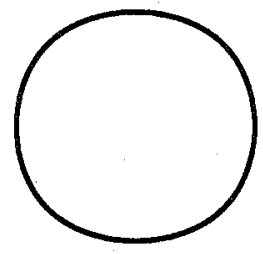

$\mathrm{t}=0.4$

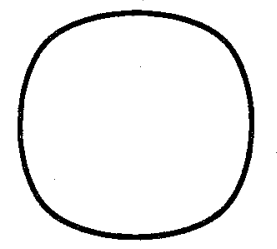

$\mathrm{t}=2.0$

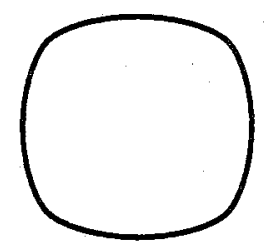

$\mathrm{t}=+\infty$

Figure 3.8: The extended velocity function is $v=r(1-\alpha \cos 4 \theta)$ with $\alpha=0.05$. Characteristics with $S_{0}$

(b) Time evolution of $\tilde{P}_{t}$

As is shown Fig.3.8(a), all the characteristics never cross each other. Thus $P_{t}$ form a surface for $t \geq 0$. Fig.3.8(b) displays a continuous deformation from $S_{0}$ to $\Xi_{v}=\partial W_{v}$.

\section{Example 8}

The function $v$ is convex but non-differentiable, which is taken from Example 4. Characteristics also do not cross. Note that characteristics radiate from the points on $S_{0}$ where $v$ is not differentiable which is indicated by round markers as shown in Fig.3.9(a).
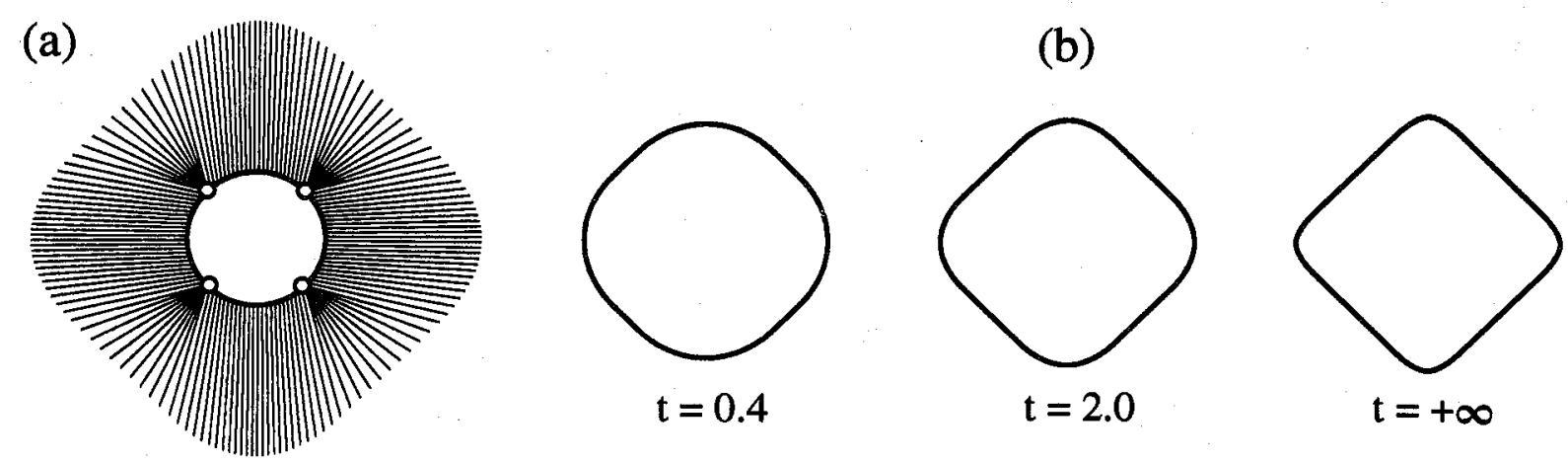

Figure 3.9: The extended velocity function is $v=r\{1+\alpha(|\cos 2 \theta|-1 / 2)\}$ with $\alpha=0.25$.
(a) Characteristics with $S_{0}$
(b) Time evolution of $\tilde{P}_{t}$

Therefore the facets emerge as soon as the growth starts, which coexist with the round 
corners all through the evolution process.

In these two examples, characteristics never cross each other, and the set $P_{t}$ is always a surface. That is true if $v$ and $S_{0}$ are both convex as assumed in these examples. However, characteristics cannot help crossing each other if $v$ is non-convex, and they might cross for non-convex $S_{0}$ even though $v$ is convex. Let us assume that $v$ is non-convex here. If the two characteristics meet at the point at the same time, the shock is formed there and the characteristics end at the point. Once the shock is formed, the set $P_{t}$ becomes to have an ear at the shock point. Let $S_{t}$ be a set which is obtained by removing ears from $P_{t}$, then it is a closed curve with corners. $S_{t}$ is a solution of (3.18) when shocks appear. Also a scaled version $\tilde{S}_{t}$ of $S_{t}$ is defined by $\tilde{S}_{t}=\frac{1}{t+1} S_{t}$, then $\tilde{S}_{0}=S_{0}$ and $\tilde{S}_{\infty}=\partial W_{v}$. The last equation guarantees that $\partial W_{v}$ is actually an asymptotic growth form.

\section{Example 9}

The function $v$ is non-convex and differentiable taken from Example 2. Characteristics cross each other as shown in Fig3.10(a). The surface $S_{t}$ is smooth and coincide with $P_{t}$ in the early stage. Then the shocks are formed at the certain time which is given by
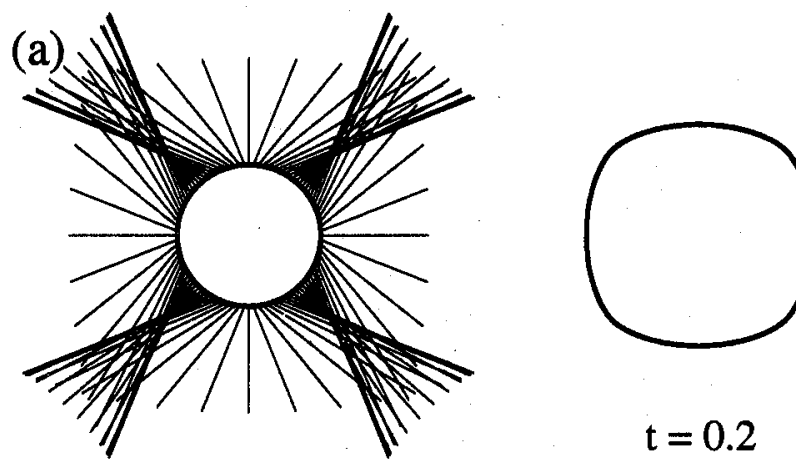

(b)

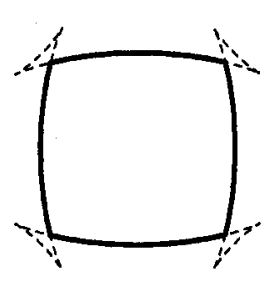

$\mathrm{t}=2.0$

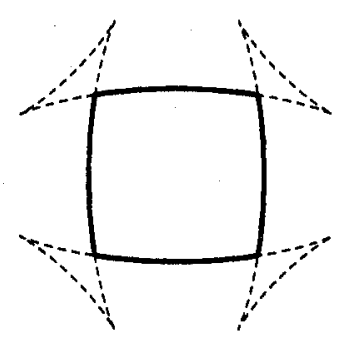

$t=+\infty$

Figure 3.10: The extended velocity function is $v=r(1-\alpha \cos 4 \theta)$ with $\alpha=0.25$.

Characteristics with $S_{0}$ (b) $\tilde{S}_{t}$ is displayed by the plain curve while $\tilde{P}_{t}$ by dashed curve.

$$
\text { Characteristics with } S_{0} \text { (b) } S_{t} \text { is displayed by the plain curve while } \tilde{P}_{t} \text { by dashed curve. }
$$

$1 /\left(\alpha-\alpha_{c}\right)$ in this example. Once the shock is formed, $S_{t}$ becomes to have corners and $P_{t}$ 
to have ears.

\section{Example 10}

The function $v$ is non-convex and non-differentiable taken from Example 5. Fig.3.11(b) shows that $S_{t}$ has both of round corners and facets in the early stage. The shocks are formed at the certain time and $S_{t}$ has corners. After a while, $S_{t}$ becomes a fully faceted square, and the form is kept from that time on.
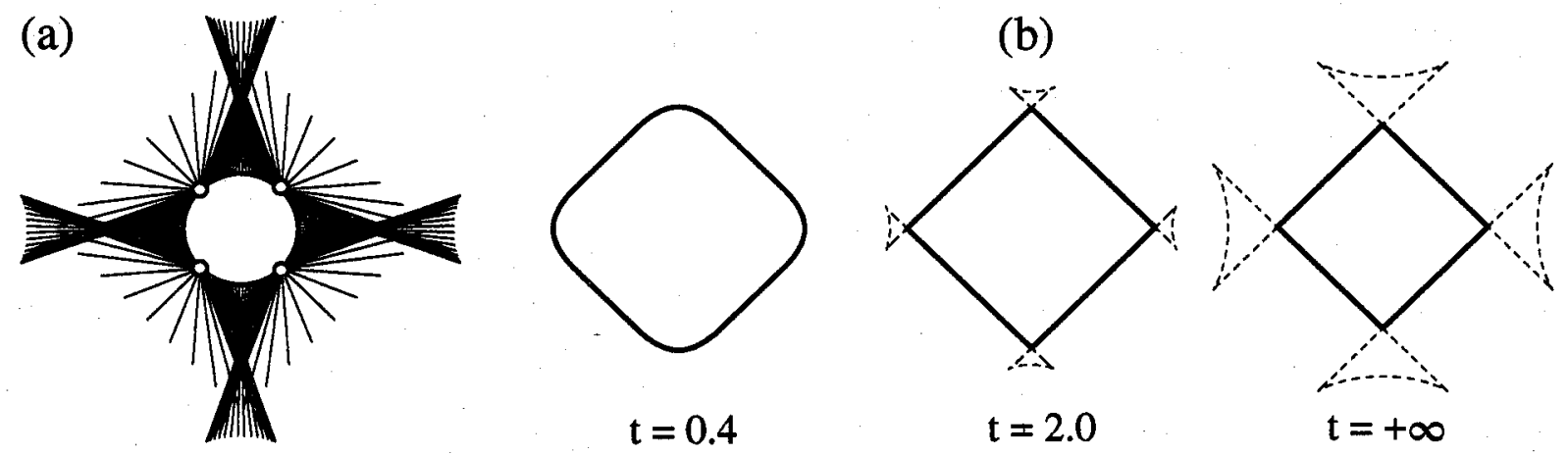

Figure 3.11: The extended velocity function is $v=r\{1+\alpha(|\cos 2 \theta|-1 / 2)\}$ with $\alpha=0.70$. $\begin{array}{ll}\text { (a) Characteristics with } S_{0} & \text { (b) } \tilde{S}_{t} \text { is displayed by the plain curve while } \tilde{P}_{t} \text { by dashed }\end{array}$ curve.

\section{Example 11}

The function $v$ is taken from Example 6. As seen in Fig.3.12(b), $S_{t}$ is an octagon with rounded corners and facets in the very early stage. Next the shocks appear and soon $S_{t}$ becomes a fully faceted octagon which is kept for a while. However the faster edges corresponding to the hidden facets disappear in a finite time, and consequently we have a fully faceted square which consists of slower edges which is a final form $\partial W_{v}$. 

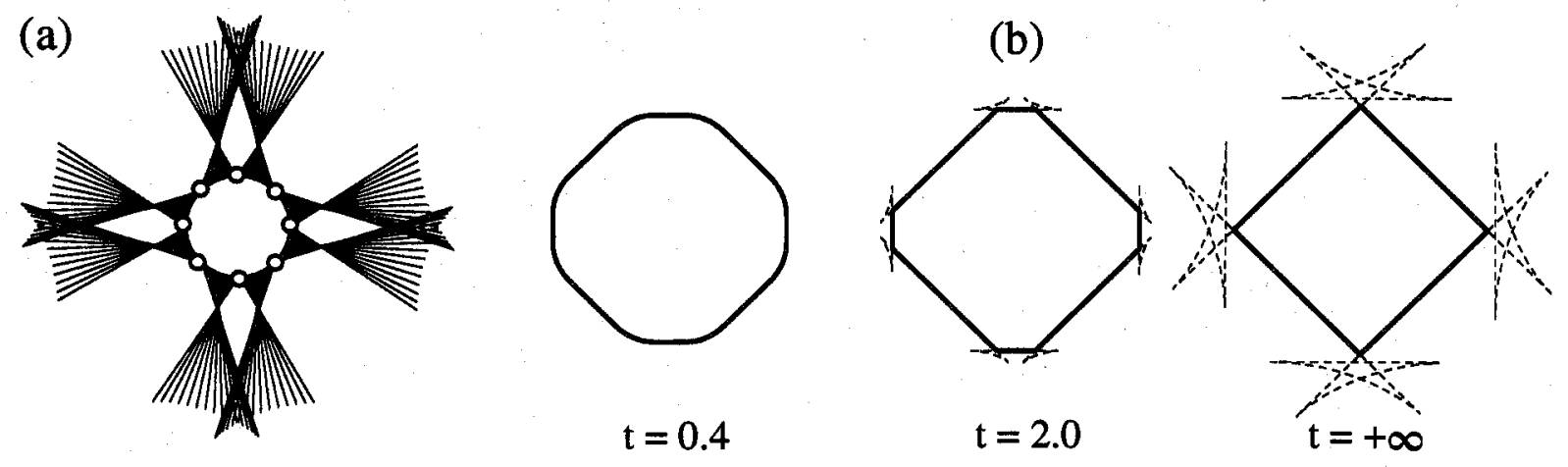

Figure 3.12: The extended velocity function is $v=r\{1+\alpha(|\cos 2 \theta|-1 / 2)+\beta(|\sin 2 \theta|-$ $1 / 2)\}$ with $\alpha=0.40$ and $\beta=0.80$.

(a) Characteristics with $S_{0}$. Characteristics emanating from the eight non-differentiable points are not drawn to avoid making the

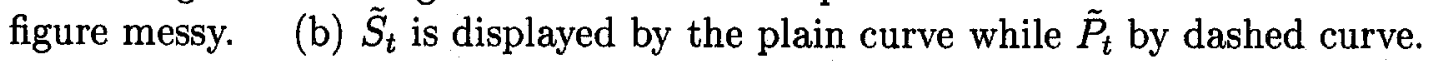

Note The method of characteristics goes back to [6]; see also [3] and [20]. The solution $S_{t}$ and $S_{t}^{a s y m}$ given in this section are considered a level set solution (see e.g. [7]) defined through the notion of viscosity solutions. 


\section{Anisotropic motion with curvature effect}

In the previous section, we neglected curvature effects and analyzed mainly the form of large scale crystals. Now we take the curvature effect into account. Throughout this section the kinetic anisotropy is assumed to give a fully faceted kinetic Wulff plot, thus the asymptotic growth form is a polygon provided that our equation includes no curvature effect. We investigate how the curvature effect give influence to the corner shape under certain situations in the 2 dimensional problems.

\subsection{Isotropic curvature effect}

Let us start with the isotropic curvature effect, i.e., $\gamma \equiv \gamma_{0}$. By defining $V_{0}=$ $\frac{c}{2}(\max \mu+\min \mu), D=\frac{V_{0} \gamma_{0}}{c}$ and $M(\theta)=\frac{c}{V_{0}} \mu(\cos \theta, \sin \theta)$, we rewrite the equation (1.1) into the following form

$$
V=M(\theta)\left(V_{0}-D \kappa\right)
$$

where $\kappa$ is a mean curvature of the surface (in 2 dimensional problems, a surface is a curve and a mean curvature is merely a curvature, of course). The sign of the curvature $\kappa$ is taken so that $\kappa=1$ for a unit circle. Note that the parameter $D$ has a dimension of diffusion constant and $M(\theta)$ is a dimensionless indicator of kinetic anisotropy. The PPHD1 function on $R^{2}$ corresponding to $M$ is also denoted by $M$ for convenience.

\section{Example 11}

Taking $M(\theta)=1+\alpha\left(|\cos 2 \theta|-\frac{1}{2}\right)$ and $\alpha$ large, the kinetic Wulff plot $\partial W_{M}$ is fully faceted as shown in Fig.4.13(a). Fig.4.13(b) presents a phase field simulation using the equation

$$
\frac{\epsilon^{2}}{D M(\theta)} \frac{\partial \phi}{\partial t}=\epsilon^{2} \nabla^{2} \phi+\phi(1-\phi)\left(\phi-\frac{1}{2}+\frac{V_{0}}{\sqrt{2} D} \epsilon\right)
$$


We just state here that the sharp interface limit of (4.23) is (4.22). In the early stage
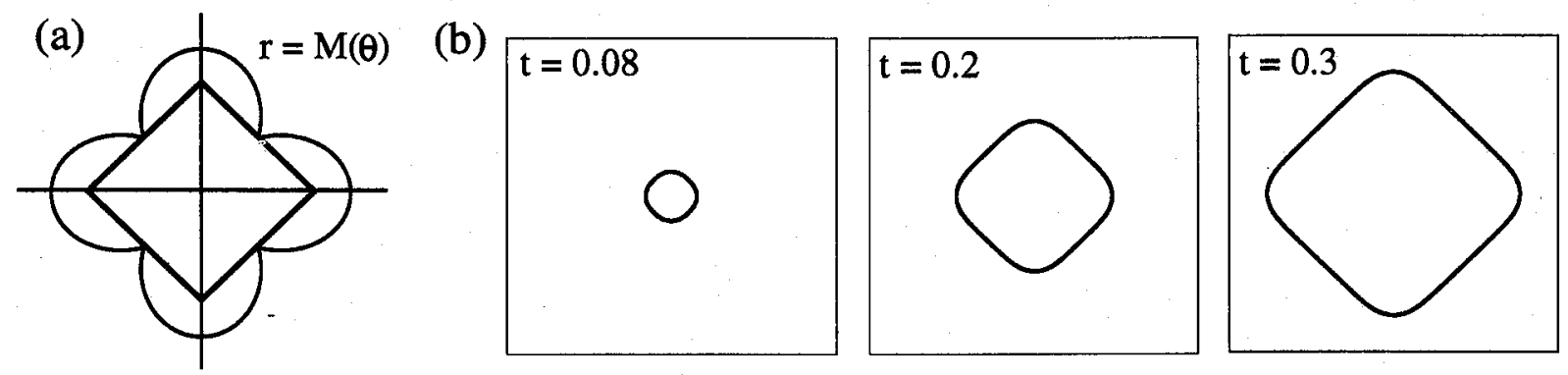

Figure 4.13: (a) Polar plot $C_{M}$ and kinetic Wulff plot $\partial W_{M}$ where $M(\theta)=1+\alpha(|\cos 2 \theta|-$ $1 / 2)$ with $\alpha=0.6$. the circular nucleus.

when the crystal is small enough, the shape is almost circular which is deformed a little by anisotropy. However, it looks to consist of growing facets and round corners with fixed size once the crystal excesses a certain size, and the curvature effect seems to be localized at the corners.

Let us examine whether facets and round corners coexist and corner rounding is localized in the later stage of growth process. Assume here that the kinetic Wulff plot $\partial W_{M}$ is a regular polygon. By rotating the coordinate system around the origin $O$ appropriately, one of the vertex of $\partial W_{M}$, say $A$, can be set on the half line $\theta=0$ as indicated in Fig.4.14(a). Let the orientations of the facets which include the vertex $A$ be $\pm \theta_{F}$ as shown in Fig.4.14(a). Set $b(\theta)=\frac{M(\theta)}{\cos \theta}$ which is a dimensionless function, then $0<b\left(\theta_{F}\right)<b(\theta)$ for $\theta \in\left(0, \theta_{F}\right)$ holds since $\partial W_{M}$ is fully faceted and no facet between the facets with the angles $\pm \theta_{F}$. Take a coordinate $s$ an arc length measured from the tip $T$ along the interface and $\theta(s)$ an argument of the outer normal vector at the point having the arc coordinate $s$ as indicated in Fig.4.14(b). Then the equation (4.22) becomes

$$
V=M(\theta)\left(V_{0}-D \frac{d \theta}{d s}\right)
$$


Assume such an interface as displayed in Fig.4.14(b) is traveling with the constant velocity $\bar{V}$ to the right keeping its profile unchanged. The point $s=S$ is a connection point between the facet and the corner. The normal velocity at the point whose normal has an angle $\theta$ is represented by $V=\bar{V} \cos \theta$. Therefore
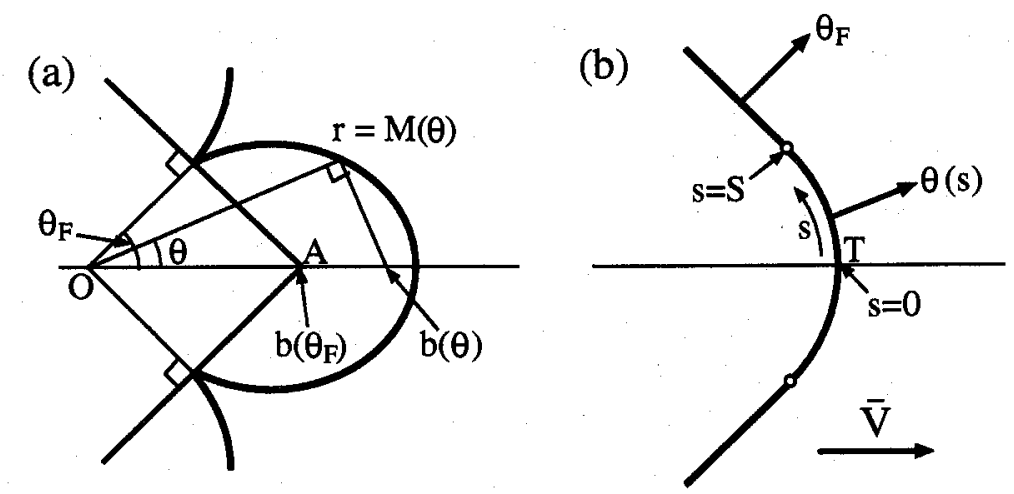

Figure 4.14: (a) $C_{M}$ and $\partial W_{M}$ between two cusps. (b) Description of the round corner. $S$ might be $+\infty$.

$$
\begin{aligned}
& M(\theta)\left(V_{0}-D \frac{d \theta}{d s}\right)=\bar{V} \cos \theta \\
& \theta(0)=0, \quad \theta(S)=\theta_{F}, \quad \frac{d \theta}{d s}(S)=0
\end{aligned}
$$

holds. Here the critical radius is defined by $R_{c}=D / V_{0}$. It is a size such that the crystal with the circular shape whose radius greater than $R_{c}$ grows while the smaller one shrinks and disappears. By substituting $s=S$, we have $b\left(\theta_{F}\right)=\frac{\bar{V}}{V_{0}}$, then $b(\theta)\left(1-R_{c} \frac{d \theta}{d s}\right)=$ $b\left(\theta_{F}\right)$. Finally we obtain

$$
\begin{aligned}
& R_{c} \frac{d \theta}{d s}=1-\frac{b\left(\theta_{F}\right)}{b(\theta)} \\
& \theta(0)=0, \quad \theta(S)=\theta_{F}
\end{aligned}
$$

which is an equation to be solved with an unknown function $\theta(s)$ and an unknown quantity $S$. We can solve the initial value problem $(4.27)$ with $\theta(0)=0$ first, then calculate $S$. Let 
us define the function $P(\theta)$ by

$$
P(\theta)=R_{c} \int_{0}^{\theta} \frac{b\left(\theta^{\prime}\right)}{b\left(\theta^{\prime}\right)-b\left(\theta_{F}\right)} d \theta^{\prime}
$$

Because $P^{\prime}(\theta)>0$ for $\theta \in\left(0, \theta_{F}\right), P(\theta)$ is invertible. Therefore $\theta(s)=P^{-1}(s)$ is a solution of (4.27) satisfying $\theta(0)=0$. Now we get $S=P\left(\theta_{F}\right)$ and the problem was solved. However, it should be examined whether $P\left(\theta_{F}\right)$ is finite or not. It is easily seen that it depends on the sharpness of the cusp of $C_{M}$ at $\theta_{F}$. Assume $M(\theta)-M\left(\theta_{F}\right)$ is of order $\left|\theta_{F}-\theta\right|^{\chi}$ in the vicinity of $\theta_{F}$, then $0<\chi \leq 1$ must hold because of the cusp shape of $M(\theta)$ at $\theta=\theta_{F}$. Thus we classify cusps into the two types, the former is given by $\chi=1$ which is called a normal cusp and the latter by $0<\chi<1$ called a sharp cusp in this article. By the formula (4.29), $S$ is finite for sharp cusps and infinite for normal. Therefore the corner formed by the curvature effect is localized if and only if the cusp is sharp. Sharp cusp means a cusp with infinitely large slope in $C_{M}$, a spine with infinitely large slope in $F_{M}$ and an infinitely long line segment for the extended $\nabla f$. If the cusp is normal, $S=+\infty$ then the corner never be localized and no facet can exists in such a traveling solution as Fig.4.14(b). Moreover there is no solution which has a flat part when the cusp is normal. This follows from the strong maximum principle [19]. If all the cusps are sharp and $\partial W_{M}$ is fully faceted, we can construct the growing form explicitly by jointing the facet parts by the corner parts given above. There is no such a solution with localized corners in the case of the normal cusps.

However, this result seems to contradict with the observation in the phase field simulations which show us the localized corners even though the anisotropy gives normal cusps. We claimed above that no facets exist for normal cusps, while we found 'facets' in our simulation. This is not a contradiction since the 'facet' is not straight in mathematical rigor but it is almost straight. Actually $\theta(s)$ converges exponentially to $\theta_{F}$ as $s$ tends to $+\infty$ for the normal cusp. 


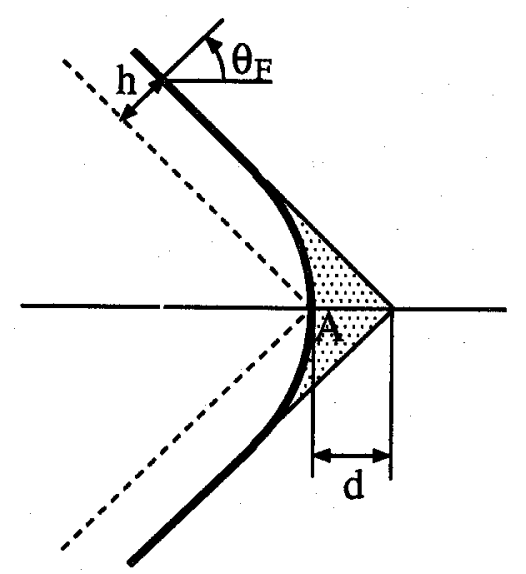

Figure 4.15: Shaded area represents a region which is rounded off from the corner.

Thus our next interest is to estimate how much the corner is rounded off by the curvature effect. Let $h$ and $d$ be distances which are indicated in Fig.4.15. The distance $h$ is calculated by

$$
h=\int_{0}^{\infty} \sin \left(\theta_{F}-\theta(s)\right) d s=R_{c} \int_{0}^{\theta_{F}} \sin \left(\theta_{F}-\theta\right) \frac{b(\theta)}{b(\theta)-b\left(\theta_{F}\right)} d \theta .
$$

This integral is always finite whichever type of cusp is concerned since the term $\sin \left(\theta_{F}-\theta\right)$ cancels out the singularity of $\left(b(\theta)-b\left(\theta_{F}\right)\right)^{-1}$ at $\theta=\theta_{F}$. And the depth of corner rounding $d$ is expressed by the formula

$$
d=\frac{h}{\cos \theta_{F}}=\frac{R_{c}}{\cos \theta_{F}} \int_{0}^{\theta_{F}} \sin \left(\theta_{F}-\theta\right) \frac{b(\theta)}{b(\theta)-b\left(\theta_{F}\right)} d \theta .
$$

This value together with the curvature radius at the tip

$$
R_{t i p}=R_{c} \frac{b(0)}{b(0)-b\left(\theta_{F}\right)}
$$

characterizes the round corner.

Let us examine the dependence of $R_{t i p}$ and $d$ on the value of $\alpha$ for the both cases of normal cusps and sharp cusps. Normal cusps are attained, for example, by setting $M(\theta)=1-\alpha\left(|\cos 2 \theta|-\frac{1}{2}\right)$. There is a critical value $\alpha_{c}^{0}$ below which the kinetic Wulff 
plot is not fully faceted, and also a critical value $\alpha_{c}^{1}$ above which the positivity of $M(\theta)$ is violated. These are given by $\alpha_{c}^{0}=0.4$ and $\alpha_{c}^{1}=2.0$ in this setting. Fig.4.16(a) plots the values of $R_{t i p}$ and $d$ against $\alpha$ in the interval $\alpha_{c}^{0}<\alpha<\alpha_{c}^{1}$. It shows that both of $R_{t i p}$ and $d$ are decreasing with respect to $\alpha$. They are of order $R_{c}$ except for the values of $\alpha$ close to $\alpha_{c}^{0}$. If we take $M(\theta)=1-\alpha\left(\sqrt{|\cos 2 \theta|}-\frac{1}{2}\right)$, the cusps are sharp. The critical values are $\alpha_{c}^{0}=2(3-2 \sqrt{2})=0.343$ and $\alpha_{c}^{1}=2.0$. The values of $R_{t i p}$ and $d$ are ploted in Fig.4.16(b) which has no much difference from Fig.4.16(a).
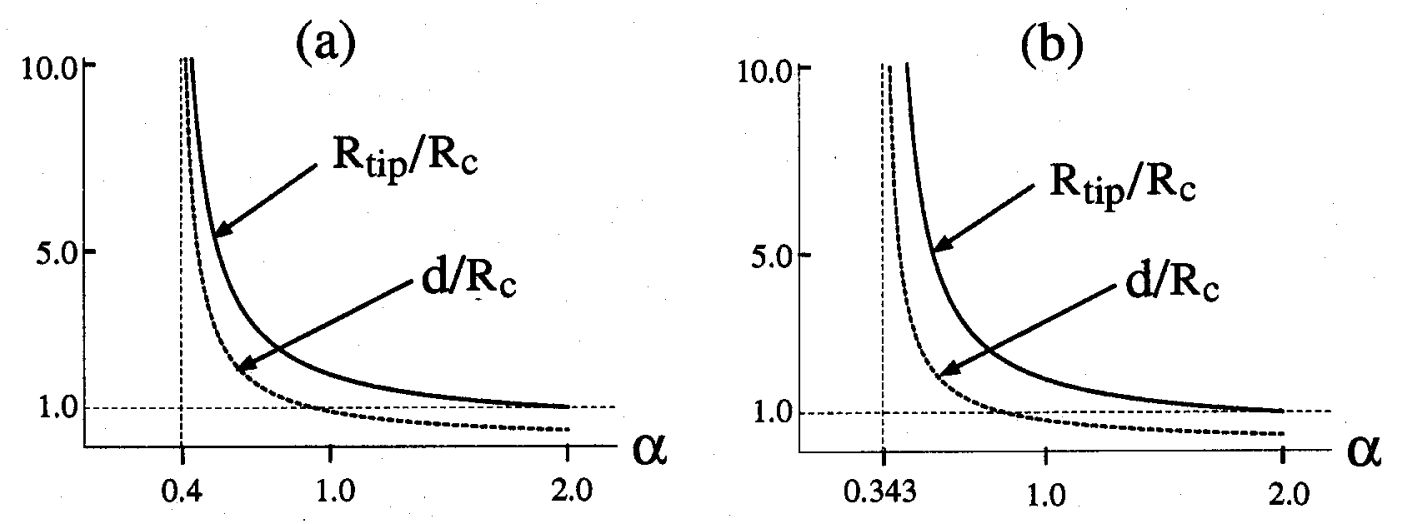

Figure 4.16: Plain curve and doted curve indecate $R_{t i p} / R_{c}$ and $d / R_{c}$ respectively, where (a) $M(\theta)=1-\alpha(|\cos 2 \theta|-1 / 2)$ and (b) $M(\theta)=1-\alpha(\sqrt{|\cos 2 \theta|}-1 / 2)$.

Let us summarize the results obtained here shortly. In mathematically rigorous sense, the curvature effect is localized at the corner only when the cusps of $C_{M}$ are sharp. However, the corner rounding is actually localized in the region with the size of order $R_{c}$ regardless of the sharpness of cusps provided that the kinetic anisotropy is strong enough. Remind that the equation (4.22) include three factors, bulk driving force, surface tension and kinetic anisotropy. The critical radius $R_{c}$ is determined by the ratio between the surface tension and the bulk driving force. And the structure scaled by $R_{c}$ is determined by the kinetic anisotropy as shown above. Therefore the size of round corners is smaller if the ratio between the surface tension and the bulk driving force is smaller and the 
anisotropy is stronger. Round corners do not change their size in the growth process once the crystal size becomes large compared with $R_{c}$. Thus the scaled asymptotic form converges to the kinetic Wulff plot which is a polygon. As was shown, $\partial W_{M}$ does not have the information of concave part of $M$. On the other hand, the corner structure mainly depend on the concave part of $M$ through the formula (4.31) and (4.32).

Note It is observed in [4] that the scaled asymptotic form converges to the kinetic Wulff shape for (1.1). Without curvature effects a rigorous proof for (3.18) is given by [23] and [18] independently. A rigorous proof is given in [22] for (1.1) when two anisotropy $\mu$ and $\gamma$ are proportional. Later it was extended for general $\mu$ and $\gamma$ in [14]. (These results actually include higher dimensional version as well.) In both works the Frank diagram $\tilde{F}_{\gamma}$ is assumed to be smooth and convex. However, the solutions studied there are general growing (level-set) solutions [7] not necessarily convex. About the finer structure of the asymptotics near the corner when $W_{M}$ is a polygon, an asymptotic formula of its curvature is given in [17]. The exact solution having round corner is given by Uwaha [27] including the equation (1.1) with interfacial anisotropy. Of course, the formula agrees with ours, however in [27] it was not discussed whether there is exact solutions having facets depending on the sharpness of the cusp of $M$. Also the formula (4.31) for $d$ is not included in [27]. In [28] the asymptotic behavior of curvature is culculated numerically by using the evolution equation of curvature functions (in the Gaussian coordinate) equivalent to (1.1). Their results are consistent with previous results and ours. Unfortunately, there is no rigorous proof of the convergence of the finer asymptotic shape reflecting the structure of the corner discussed in the previous paragraph. 


\subsection{Anisotropic curvature effect}

We next examine the anisotropic curvature effect. Let us express the anisotropic surface free energy density by $\gamma(\boldsymbol{n})=\gamma_{0} G(\boldsymbol{n})$ where $G(\boldsymbol{n})$ is a dimensionless indicator of anisotropy. Because we are concerned with the 2 dimensional problems, $G$ is given in the form $G(\theta)$, while $G$ is also used for the extended PPHD1 function as well as the usage of $M$. Then the equation of interfacial motion is written in the form

$$
V=M(\theta)\left(V_{0}-D \kappa_{G}\right)
$$

where $\kappa_{G}$ is a weighted mean curvature with respect to the dimensionless surface free energy $G$. The kinetic Wulff plot $\partial W_{M}$ is assumed to be fully faceted as well as before. Here we restrict our interest to the case where the Wulff plot $\partial W_{G}$ also has facets.

\section{Example 12}

The anisotropies are given by $M(\theta)=1+\alpha\left(|\cos 2 \theta|-\frac{1}{2}\right)$ and $G(\theta)=1+\beta\left(|\sin 2 \theta|-\frac{1}{2}\right)$. The value of $\alpha$ is taken to be greater than the critical value 0.4 , and $\beta$ less than that . Therefore $\partial W_{M}$ is fully faceted and $\partial W_{G}$ is partially faceted as shown in Fig.4.17(a). Also Fig.4.17(a) indicates that the orientations of facets are different by $\frac{\pi}{4}$ between $\partial W_{M}$ and $\partial W_{G}$. Physically speaking, this situation is somewhat unrealistic since the cusps in both type of anisotropies usually appear at the angle corresponding to the singular interface along which the molecules are densely packed. However, it might be useful to examine this example since it makes the relation between the two anisotropies clear in mathematical sense. Fig.4.17(b) shows a phase field simulation using the eqation

$$
\frac{\epsilon^{2} G(\theta)}{D M(\theta)} \frac{\partial \phi}{\partial t}=\epsilon^{2} \nabla \cdot\left[G(\theta)\left(G(\theta) I+G^{\prime}(\theta) J\right) \nabla \phi\right]+\phi(1-\phi)\left(\phi-\frac{1}{2}+\frac{V_{0}}{\sqrt{2} D} \epsilon\right)
$$

where $I$ is the identity matrix and $J$ is the $\pi / 2$-rotation matrix. In the practical simulations, the modulus function $|p|$ in $G(\theta)$ is approximated by $\frac{1}{\rho} \ln \cosh \rho p$ with large positive 
(a)

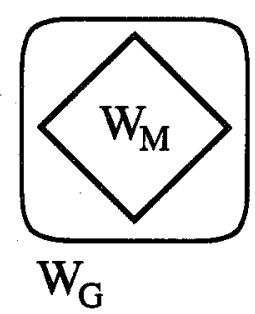

(b)

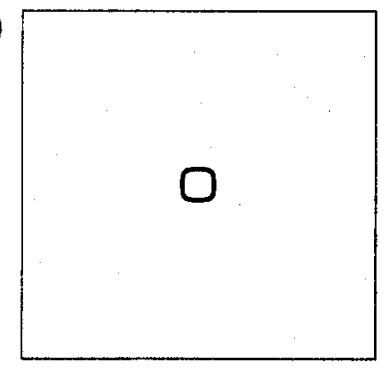

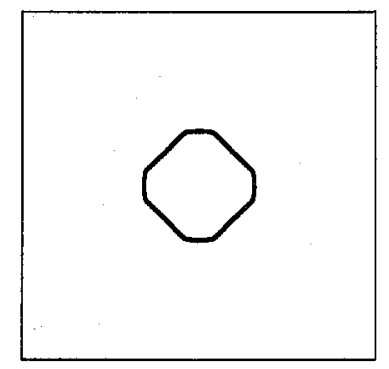

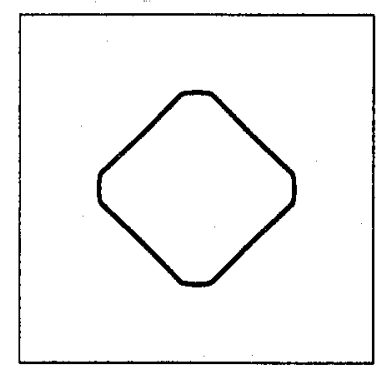

Figure 4.17: (a) Kinetic Wulff set $W_{M}$ and Wulff set $W_{G}$ where $M(\theta)=1+$ $\alpha\left(|\cos 2 \theta|-\frac{1}{2}\right)$ and $G(\theta)=1+\beta\left(|\sin 2 \theta|-\frac{1}{2}\right)$ with $\alpha=1.0$ and $\beta=0.25$. Corresponding phase field simulation of the growth process from the circular nucleus.

$\rho$. In the early stage when the crystal is small, the crystal form is almost similar to $\partial W_{G}$, which implies that the curvature effect dominates the kinetic one. However, soon the crystal exhibit an octagonal shape by the conbination of two types of anisotropies. The corner is cut from the growing kinetic Wullf plot by the fixed size in the later growth process. It is similar to Example 11 where the corner is rounded from the growing kinetic Wullf plot by the fixed size.

From this result we are motivated to construct a traveling corner solution similar to the one calculated in the previous subsection. Before doing that the meaning of the weighted mean curvature $\kappa_{G}$ has to be made clear. As long as the indicator function $G(\theta)$ is twice differentiable, $\kappa_{G}$ is written by the form

$$
\kappa_{G}=\left(G(\theta)+G^{\prime \prime}(\theta)\right) \kappa,
$$

where $\kappa$ is an usual (mean) curvature. If $G(\theta)$ has a cusp, it seemes that the facet appears with the corresponding orientation from the simulation. At the cusp angle, $G^{\prime \prime}(\theta)$ can be regarded as $\Delta G^{\prime} \cdot \delta$ where $\Delta G^{\prime}$ is a gap of $G^{\prime}(\theta)$ and $\delta$ is a delta function at the angle. Thus $\kappa_{G}$ has a form " $+\infty \times 0$ ", and we can expect that it is finite and still has some physical meaning. Fortunately it is true $[24,12]$. It is evaluated through the variational 
interpretation of surface free energy, which is

$$
\kappa_{G} \simeq \frac{\Delta \text { Energy }}{\Delta \text { Volume }}
$$

where $\Delta$ Energy is a variation of dimensionless surface free energy $G$ against the small volume change. Fig.4.18(a) represents a horizontal facet with energy density $G_{0}$ and adjacent two facets whose orientation is indicated by $\varphi_{1}$ and $\varphi_{2}$ and whose energy density are $G_{1}$ and $G_{2}$ respectively. Consider the situation that the horizontal facet moves upward

(a)

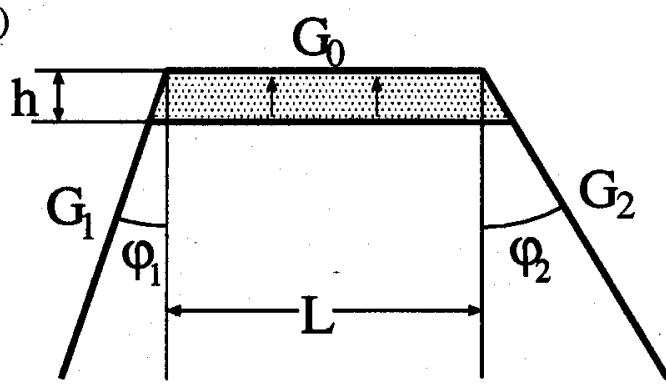

(b)

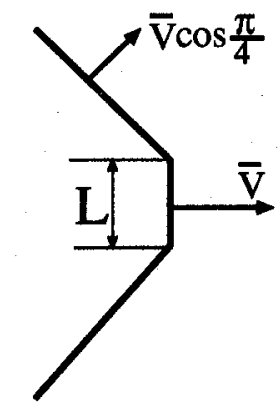

Figure 4.18: (a) Graphical derivation of weighted mean curvature.

(b) Traveling corner solution.

by the distance $h$. Then $\Delta$ Volume $\simeq L h$ and $\Delta$ Energy $\simeq \frac{G_{1} h}{\cos \varphi_{1}}+\frac{G_{2} h}{\cos \varphi_{2}}-G_{0}\left(\tan \varphi_{1}+\right.$ $\left.\tan \varphi_{2}\right) h$. Therefore

$$
\kappa_{G}=\lim _{h \rightarrow 0} \frac{\Delta \text { Energy }}{\Delta \text { Volume }}=\frac{1}{L}\left\{\frac{G_{1}}{\cos \varphi_{1}}+\frac{G_{2}}{\cos \varphi_{2}}-G_{0}\left(\tan \varphi_{1}+\tan \varphi_{2}\right)\right\} .
$$

Note that the orientations of two slanting surfaces need not correspond to the cusps of $G$; in such a case $\varphi_{i}$ and $G_{i}(i=1,2)$ are evaluated as the limiting value at the corner. From (4.37), it is easily seen that $\kappa_{G}$ can be non-zero on the flat surface at which the usual curvature $\kappa$ vanishes. It should be noticed such a situation occurs only when the flat surface is a facet induced by the cusp of $G$. On the other hand, $\kappa_{G}$ must vanish on the flat surface which is not derived from the cusp of $G$. 
Let us apply (4.37) to example 12 to construct a traveling corner solution which moves to the right in the constant velocity $\bar{V}$ as indicated in Fig.4.18(b). The slanted facets $(\theta= \pm \pi / 4)$ does not correspond to the cusp of $G$ and thus $\kappa_{G}=0$ there. Thus $\bar{V} \cos \frac{\pi}{4}=V_{0} M(\pi / 4)$ holds. On the other hand the facet at the tip $(\theta=0)$ comes from the cusp of $G$, and $\kappa_{G}$ there is evaluated by $\kappa_{G}=\frac{2}{L}(\sqrt{2} G(\pi / 4)-G(0))$ by setting $\varphi_{1}=\varphi_{2}=\pi / 4$ in (4.37). Therefore $\bar{V}=M(0)\left\{V_{0}-\frac{2 D}{L}(\sqrt{2} G(\pi / 4)-G(0))\right\}$. Solving these equations we get $\bar{V}=V_{0} b(\pi / 4)$ and $L=2 R_{c} \frac{b(0)}{b(0)-b(\pi / 4)}(\sqrt{2} G(\pi / 4)-G(0))$ where $b(\theta)=\frac{M(\theta)}{\cos \theta}$. Then $L$ is of order $R_{c}$ as well as the corner rounding estimated in the previous subsection.

\section{Example 13}

At last, we make $\partial W_{M}$ and $\partial W_{G}$ fully faceted squares and having same orientation as shown in Fig4.19(a). It is attained by setting $M(\theta)=1+\alpha\left(|\cos 2 \theta|-\frac{1}{2}\right)$ and $G(\theta)=1+\beta\left(|\cos 2 \theta|-\frac{1}{2}\right)$, and taking $\alpha$ and $\beta$ large. Fig4.19(b) is a corresponding phase field simulation, which displays a growing square solution. Taking the edges' length

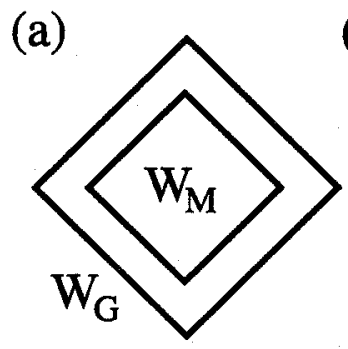

(b)
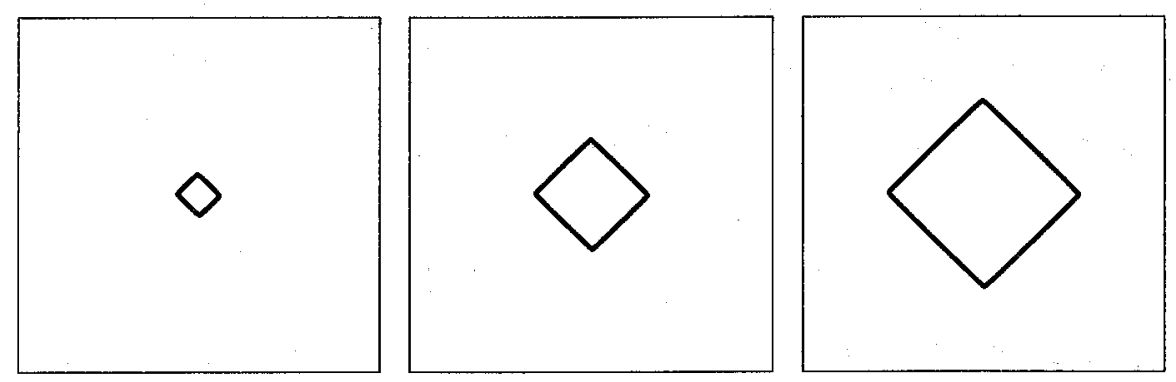

Figure 4.19: (a) Kinetic Wulff set $W_{M}$ and Wulff set $W_{G}$ where $M(\theta)=1+$ $\alpha\left(|\cos 2 \theta|-\frac{1}{2}\right)$ and $G(\theta)=1+\beta\left(|\cos 2 \theta|-\frac{1}{2}\right)$ with $\alpha=1.50$ and $\beta=0.60$. Corresponding phase field simulation of the growth process from the circular nucleus. 
$L(t)$ and using the formula (4.37) with $\varphi_{1}=\varphi_{2}=0$, the equation (4.33) becomes

$$
\frac{d L}{d t}=M_{0}\left(V_{0}-\frac{2 D G(\pi / 4)}{L}\right) \text {, }
$$

where $M_{0}$ is the value of $M$ at the cusp point. There is a critical size $L_{c}=\frac{2 D G(\pi / 4)}{V_{0}}$ in this type of solution expressing the square crystal. If the initial shape is square and the initial size $L_{0}$ is greater than $L_{c}$, the solution keep on growing and soon converges to the asymptotic growth whose growth rate is $M_{0} V_{0}$. If $L_{0}$ is smaller than $L_{c}, L(t)$ vanishes in finite time. In this example, curvature effect does not change the corner shape of $\partial W_{M}$ since its corner shape is the one which is prefered by the anisotropic curvature effect.

The solutions presented in this section have rounded (or cut) corners whose size is fixed to the order $R_{c}$ in the later stage of growth process, except for the cases like Example 13. On the other hand, such kind of corners do not appear in the solution of $V=v(\boldsymbol{n})$ as was shown in the previous section. Although its solution might exhibit round corners as shown in Fig.3.9, the surface is magnified keeping its shape in the later stage of evolution, thus the size of round corners is also magnified. Also Fig.3.12 apparently resembles Fig.4.17. However, they are totally different. The crystal form in Fig.3.12 can be an octagon only temporally and change to the square in a finite time. On the other hand, Fig.4.17 shows a solution which is an octagon forever, while its scaled shape converges to the square.

Note The equation (1.1) with singular interfacial energy $\gamma$ was first studied by J.Taylor and S.Angenent-M.Gurtin more than a decade ago. The reader is referred to the article [11] in this volume for the developement of the theory as well as [24],[12] and [8]. For a review of the equation with singular diffusivities, the reader is referred to [15] and [10]. We note that it is quite recent that a level set motion of solution was established for (1.1) with singular $\gamma$ [9]. It turns out that the scaled asymptotic form converges to the kinetic Wulff shape $W_{M}$ even $\gamma$ is singular. As noted in [8], once a level set method has been established, such a statement can be proved as in [14]. Phase field model including 
anisotropy is studied in [16] and [25]. See also [8] and [9] and the references cited there for recent development of its mathematical analysis.

Acknowledgement YG was partially suported by a Grant-in-Aid for Scientific Research, No.10304010, 12874024 the Japan Society of the Promotion of Science. 


\section{References}

[1] J. W. Cahn and W. C. Carter, Met. Trans., vol. 27A (1996), 1431-1440. (Also published in Selected Works of John Cahn.)

[2] J. W. Cahn and D. W. Hoffman, Acta Metall., vol.22 (1974), 1205-1214.

[3] J. W. Cahn, J. E. Taylor and C. A. Handwerker, Sir Charles Frank, OBE, FRS: An eighties birthday tribute, Eds. R. G. Chambers, J. E. Enderby, A. Keller, A. R. Lang and J. W. Steeds, Adam Hilger, Bristol, Philadelphia and New York (1991) p.88.

[4] A. A. Chernov, Kristallografia, vol. 7 (1962), 895. (Soviet Phys. Cryst., vol. 7 (1963), 728.)

[5] F. H. Clarke, Optimization and Nonsmooth Analysis, Canadian Math. Soc. Ser. of Monographs and Advanced Texts, John Wiley \& Sons, New York (1983).

[6] F. C. Frank, Growth and Perfection of Crystals, Eds. R. H. Doremus, B. W. Roberts and D. Turnbull, John Wiley, New York (1958), p.411.

[7] Y. Giga, Sugaku, vol. 47 (1995), 321-340; English transl. Sugaku Expositions, vol. 10 (1997), 217-241.

[8] Y. Giga, Sugaku, vol. 52 (2000), 113-127 (in Japanese), English translation in preparation.

[9] M. -H. Giga and Y. Giga, Generalized motion by nonlocal curvature in the plane, preprint.

[10] M. -H. Giga, Y. Giga and R. Kobayashi, Proc. Taniguchi Conf. on Mathematics, Nara 98, ed. T.Sunada, pp.1-34 (2000)

[11] Y. Giga, M. Paolini and P. Rybka, JJIAM, this volume to appear. 
[12] M. E. Gurtin, Thermomechanics of Evolving Phase Boundaries in the Plane, Oxford, Clarendon Press (1993).

[13] D. W. Hoffman and J. W. Cahn, Surf. Sci., vol.32 (1972), 368-388.

[14] H. Ishii, G. E. Pires and P. E. Souganidis, J. Math. Soc. Japan, vol. 51 (1999), 267.

[15] R. Kobayashi and Y. Giga, J. Stat. Phys., vol. 95 (1999), 1187-1220.

[16] G. B. McFadden, A. A. Wheeler, R. J. Braun, S. R. Coriell and R. F. Sekerka, Phys. Rev. E, vol. 48 (1993), 2016-2024.

[17] H. Muller-Krumbhaar, T. W. Burkhardt and D. M. Kroll, J. Crystal Growth, vol. 38 (1977), 13.

[18] S. Osher and B. Merriman, Asian J. Math., vol. 1 (1977), 560-571.

[19] M. H. Protter and H. F. Weinberger, Maximum Principles in Differential Equations, Englewood Cliffs, N.J., Prentice Hall (1967).

[20] A. Pimpinelli and J. Villain, Physics of Crystal Growth, Cambridge University Press (1997)

[21] R. Schneider, Convex Bodies : The Brunn-Minkowski Theory, Cambridge University Press, Cambridge (1993).

[22] H. M. Soner, J. Differential Equations, vol. 101 (1993), 313.

[23] P. Soravia, Nonlinear Analysis, TMA, vol. 22 (1994), 1247-1262.

[24] J. E. Taylor, Acta Metall., vol.40 (1992), 1475-1485.

[25] J. E. Taylor and J. W. Cahn, Physica D, vol. 112 (1998), 381-411. 
[26] J. E. Taylor, J. W. Cahn and C. A. Handwerker, Acta Metall., vol.40 (1992), 14431474.

[27] M.Uwaha, J. Crystal Growth, vol. 80 (1987), 84-90.

[28] E. Yokoyama and R. F. Sekerka, J. Crystal Growth, vol.125 (1992), 389-403. 


\section{Appendix}

Appendix 1 The of PHD1 functions (2.4) - (2.6)

Proof: Differentiating $f(\lambda \boldsymbol{x})=\lambda f(\boldsymbol{x})$ by $\boldsymbol{x}$ we get (2.4). Differentiating $f(\lambda \boldsymbol{x})=\lambda f(\boldsymbol{x})$ by $\lambda$ and setting $\lambda=1$ yields (2.5). Taking the exterior derivative of the both sides of (2.5) we have $d f(\boldsymbol{x})=d \boldsymbol{x} \cdot \nabla f(\boldsymbol{x})+\boldsymbol{x} \cdot d \nabla f(\boldsymbol{x})$. From $d f(\boldsymbol{x})=\nabla f(\boldsymbol{x}) \cdot d \boldsymbol{x}$ the identity (2.6) follows.

Appendix 2 Equivalence of (1) - (3) in the section 2.2

Proof: It is obvious that $(1) \longrightarrow(3)$ and $(2) \longrightarrow(1)$. Therefore it is enough to show (3) $\longrightarrow(2)$. Let us take arbitrarily $\boldsymbol{x}$ and $\boldsymbol{y}$ which are not $\mathbf{0}$. Take $\boldsymbol{\xi}=\frac{\boldsymbol{x}}{f(\boldsymbol{x})}$ and $\boldsymbol{\eta}=\frac{\boldsymbol{y}}{f(\boldsymbol{y})}$, then $\boldsymbol{\xi}$ and $\boldsymbol{\eta}$ belong to the set $A=\{\boldsymbol{x} ; f(\boldsymbol{x}) \leq 1\}$ (actually belong to its boundary).

$$
f(\boldsymbol{x}+\boldsymbol{y})=f(f(\boldsymbol{x}) \boldsymbol{\xi}+f(\boldsymbol{y}) \boldsymbol{\eta})=(f(\boldsymbol{x})+f(\boldsymbol{y})) f(\boldsymbol{\zeta})
$$

where $\boldsymbol{\zeta}=\frac{f(\boldsymbol{x})}{f(\boldsymbol{x})+f(\boldsymbol{y})} \boldsymbol{\xi}+\frac{f(\boldsymbol{y})}{f(\boldsymbol{x})+f(\boldsymbol{y})} \eta$. The point $\boldsymbol{\zeta}$ belongs to $A$, since it lies on the line segment connecting $\boldsymbol{\xi}$ and $\boldsymbol{\eta}$. Therefore $f(\boldsymbol{\zeta}) \leq 1$ and $f(\boldsymbol{x}+\boldsymbol{y}) \leq f(\boldsymbol{x})+f(\boldsymbol{y})$ which was the inequality to be proved.

Appendix 3 A subadditive PHD1 function is called a sublinear function. For a subset $K$ of $R^{n}$ let $h_{K}$ denote the support function of $K$, i.e.,

$$
h_{K}(\boldsymbol{x})=\sup _{y \in K} \boldsymbol{x} \cdot \boldsymbol{y}
$$

which is always sublinear if it is well-defined in $R^{n}$. Let $K^{\circ}$ denote the polar body of $K$, i.e.,

$$
K^{\circ}=\left\{x \in R^{n} ; h_{K}(\boldsymbol{x}) \leq 1\right\}
$$

For the PPHD1 function $f$ we see that the Wulff set is the polar body of the Frank diagram, i.e., $W_{f}=\left(\tilde{F}_{f}\right)^{\circ}$. By definition $K^{\circ}=\bar{K}^{\circ}$ for convex hull $\bar{K}$ of $K$. Thus 
$W_{f}=W_{\bar{f}}$ where $\bar{f}$ denotes the convexification of $f$. There is a reflexibity. The polar body $W_{f}^{\circ}$ of $W_{f}$ equals to the convex hull of $\tilde{F}_{f}$. The function $\bar{f}$ is a support function of $W_{f}$. For more details on convex functions of sets, the readers are referred to a book of Schneider [21]. 